\title{
Self-Evaluation Tool for Action in Partnership: Translation and Cultural Adaptation of the Original Quebec French Tool to Canadian English
}

\author{
Angèle Bilodeau \\ Université de Montréal \\ Gillian Kranias \\ Health Nexus
}

\begin{abstract}
This article presents the translation and cultural adaptation, into Canadian English, of the Outil diagnostique de l'action en partenariat, a tool widely used to support the practice of partnerships since its creation in French, in Quebec, in 2008. The theoretical foundations and properties of the original tool are presented, followed by a summary of methodological guidelines and a description of the process and results. The methodology involved an expert committee to formulate the English tool and verify its equivalence with the original, and a pretest with target users. This rigorous procedure ensures equivalence of the translated tool and its cultural adaptation to the intended users.
\end{abstract}

Keywords: action in partnership, action network, inter-sectoral action, partnership self-evaluation tool, translation-adaptation of measurement tools

Résumé : Cet article présente la traduction et l'adaptation culturelle, en anglais canadien, de l'Outil diagnostique de l'action en partenariat, largement utilisé pour soutenir la pratique des partenariats depuis sa création en français, au Québec, en 2008. Les fondements théoriques et les propriétés de l'outil original sont présentés. Suivent ensuite la démarche méthodologique et la présentation des résultats. La méthodologie comprend un comité d'experts pour la traduction de l'outil vers l'anglais et la vérification de son équivalence avec l'outil original, et un pré-test effectué auprès d'utilisateurs potentiels. Cette procédure rigoureuse assure l'équivalence de l'outil traduit et son adaptation culturelle aux utilisateurs visés.

Mots clés : action en partenariat, action en réseau, action intersectorielle, outil d'auto-évaluation des partenariats, traduction-adaptation d'outils de mesure

Partnership action is a widely recommended strategy for addressing social determinants of complex issues at varying levels of public action (WHO, 2011). The

Corresponding author: Angèle Bilodeau, École de santé publique, Université de Montréal, Montréal, Québec, Canada, 7101 avenue du Parc, Montréal, QC H3C 3J7; angele.bilodeau@ umontreal.ca 
availability of evidence-based and user-friendly tools to evaluate the quality of partnerships is a significant lever to undertake action in partnership or follow its progress (Fetterman \& Wandersman, 2007; Halliday, Asthana, \& Richardson, 2004; Newcomer, Hatry, \& Wholey, 2015). The Self-evaluation tool for action in partnership ${ }^{1}$ is a tool widely used in Quebec in its original French version. It was developed in 2008 by researchers from the Montreal Directorate of Public Health and the Canada Research Chair Approches communautaires et inégalités de santé of l'Université de Montréal. It met the need for evaluation tools theoretically based on empirical research, was rigorously validated, and was able to fit into the current activities of partnerships. Its Canadian English adaptation meets the needs of practitioners and researchers, responding to a request from Health Nexus in Ontario. This article first details the characteristics of the original tool. Then it summarizes the methodological guidelines for the translation and validation of measurement tools. Finally, it presents the methodology and results of the translation and adaptation of the original tool to the context of English Canada.

\section{THE ORIGINAL TOOL: THE OUTIL DIAGNOSTIQUE DE L'ACTION EN PARTENARIAT}

The tool fits within a theory-based evaluation approach (Devaux-Spatarakis, 2014). More specifically, it meets the need for higher-level theories of changein contrast to local theories - which may result from multi-site analysis, metaanalysis, or the use of general social theories to produce more general interpretive models (Auspos \& Kubisch, 2004; Barnes, Matka, \& Sullivan, 2003; Chircop, Bassett, \& Tatlor, 2015; Dahler-Larsen, 2001; Villeval et al., 2016). The tool operationalizes a mid-range theory that defines six requirements for effective partnership work from a series of case studies based on Actor Network Theory (Bilodeau, Galarneau, Fournier, \& Potvin, 2011). It makes it possible to assess the functioning of a partnership, identifying both difficulties and aspects that work well with reference to the six requirements for effectiveness. It is also a self-evaluation tool, allowing practitioners to situate their own partnership according to three levels of achievement for each requirement and to make a judgment founded on empirically established best practices based on case studies.

The first four requirements are related to participation dynamics, that is, setting actors into motion to address a situation that is deemed problematic. (1) The range of perspectives relevant to the issue: Partnerships must include the various perspectives on the issue of interest to broaden the opportunities for convergence among actors. (2) Early stakeholder involvement in strategic decisions: Partners need to be actively involved in analyzing issues and developing options for action, not just in implementation. (3) Engagement of stakeholders in negotiating and influencing decisions: Partners need to be engaged to a degree that goes beyond consultation and must have real influence on decisions. (4) Commitment of strategic and pivotal stakeholders to the project: Stakeholders must be in a position to make decisions and commit resources, and essential resources for successful 
action must be mobilized. Furthermore, partners must be stable and able to bring in new stakeholders needed to move forward in action together.

The fifth and sixth requirements relate to partnership arrangements. Such arrangements concern structures and mechanisms that build cooperation and support the resolution of controversies, usually linked to questions of power or paradigms of action. (5) Partnership arrangements that favour equalization of power among the stakeholders: Favourable arrangements are structures and mechanisms that reduce subordination resulting from structural inequality among actors. Thereby, actors can identify concrete indicators of their influence and interdependence, such as the equitable acknowledgement and distribution of benefits, equal consideration of all points of view in discussion and decision, and negotiated criteria for accountability. (6) Partnership arrangements that help build collective action: These are exchanges of diverse points of view that expand possibilities for action, open up discussions over disagreements, and support their successful resolution, going beyond individual interests to find common ground in the interest of the target population and modifying individual actions to build new and more holistic options for shared action. Through such arrangements, actors can concretely identify progress in thinking and action that result from linking partner paradigms and resources.

The tool includes 18 items relating to the six requirements described above. For each item, respondents must choose one of three options representing varying degrees of achievement of the item (strong, moderate, weak) in their partnership. The tool is designed to be used by members of a partnership who voluntarily participate in self-evaluation. Three types of partnership evaluation may be produced: (1) A one-time evaluation can identify the strong/weak items or the strong/ weak requirements. The items or requirements that receive the largest number of "strong" responses indicate the strengths of the partnership. Those receiving the largest number of "weak" responses indicate its weaknesses. The items lacking consensus indicate possible areas of controversy. The items left blank indicate that some respondents are unable to assess important aspects of their partnership. (2) Longitudinal evaluation is possible if the exercise is repeated after a period of time. An evaluation performed for each item or each requirement can show improvement or deterioration over time. Finally, (3) a summary portrait can be produced across multiple partnerships within a similar context (e.g., same funder, same issue, or same policy). If more than one partnership is experiencing difficulties with the same items or requirements, this could highlight collective issues.

The original French tool was validated using three methods (Bilodeau et al., 2011). The cognitive interview (Presser et al., 2004) with target user groups served to pre-test the tool in terms of their understanding of items and response choices (first method). This is a participatory pretest method of a questionnaire where participants verbalize their understanding of the questions and answer choices. Two group interviews of five and six participants were conducted by an interviewer specialized in the field. Four items were corrected by the researchers to ensure that they were understood by the target users in the sense of the theoretical 
model. The group interviews also allowed verification of the ecological validity of the tool (second method) (Brewer, 2000), that is, whether the items and the response choices make sense in the context in which the target users work. Results confirmed that the tool worked properly for partnerships which, beyond providing a space for information sharing and networking, are the venue for collaborative work on a specific project with resources. Finally, the construct validity of the tool (third method) was tested with 28 partnerships totalling 272 respondents. Results were three-fold. First, the tool was able to capture variations in assessments: of the 4,682 responses ( 272 respondents multiplied by 18 items in the tool), $7.8 \%$ indicated conditions not met, $29.1 \%$ conditions only partially met, and $63 \%$ conditions fully met. Second, the tool allowed for a good convergence between respondents within a same partnership (intra-class correlation coefficient $=0.198$, $p<0.001)$. Third, the tool was suitable for distinguishing between partnerships with better and worse results $(F(27 ; 244)=3,38 ; p<0.001)$ and helped identify partnerships encountering difficulties with certain conditions.

\section{METHODOLOGICAL GUIDELINES FOR THE TRANSLATION AND VALIDATION OF THE MEASUREMENT TOOLS}

The relevant methodological guidelines are drawn from the rules issued by international authorities in this field (ITC, 2017; PISA, 2018; WHO, n.d.). They also come from Quebec scientific literature where expertise has developed because of the widespread use of American measurement instruments requiring translation into French and adaptation to the Quebec context. The methodology established by Vallerand in 1989 constitutes an important reference. These guidelines have been developed mainly in the fields of psychology and education.

Before a measurement tool can be used in a cultural context different from its original context, translation and cultural adaptation are required in order to claim to provide relevant results. This is for linguistic reasons, but also so that the translated tool is as natural and appropriate as the original tool and can work in the same way (ITC, 2017; WHO, n.d.). A verification of the metric properties of the translated tool is also necessary to ensure that it meets quality standards equivalent to the original tool (ITC, 2017). There is thus a distinction between the methodologies for translation and cultural adaptation of the tools, which are detailed here, and those for validation of the translated tools, which we discuss only in terms of their relevance, given the object of the present article.

\section{Translation and Cultural Adaptation of the Measurement Tools}

The objective of translation is to ensure equivalence between the translated tool and the original tool. Since it is difficult to match words from one culture to another, given the vocabulary and grammar specific to each language, equivalence of meaning rather than literal translation is targeted (ITC, 2017; PISA, 2018; WHO, n.d.). Caron (1999) distinguishes between equivalence of expressions, retaining terms and expressions specific to the target culture; experiential equivalence, 
choosing situations appropriate to the target culture; and conceptual equivalence between the specialized terms of the original tool and the translated tool. Methodologies include translation techniques and pre-testing the draft version with its target users.

Three translation techniques are commonly used: traditional translation, reverse translation, and translation committee. Traditional translation is done by a single bilingual person, the researcher, or a professional translator. This technique alone is not recommended due to the linguistic or comprehension biases inherent to a single translator (ITC, 2017; Vallerand, 1989).

Reverse translation (or back-translation) includes a first translation from the original language into the target language, then a second translation from the translated version into the original language, followed by a comparison of the two versions in the original language, leading to any necessary adjustments. One or two reverse translations can be done in parallel. This technique reduces the risk of bias due to a single translator. The accuracy of the translation in the target language is assessed by the degree to which it accurately reproduces the original version. In this way, gaps in the translation can be identified and corrected. Translators are either professional translators or specialists in the field of the tool who are bilingual and/ or familiar with the culture and linguistic characteristics of the target environment (Ouellet, 2008). Adjustments can be identified jointly by the translators (Ouellet, 2008) or by using the committee method described below (Vallerand, 1989). In practice, this method has shown that it is difficult to obtain a perfect equivalence between the translated version and the original version (Caron, 1999). In addition, by not focusing on the target language version of the tool, adaptation problems may be overlooked (Hambleton \& Patsula, 1999; ITC, 2017). As such, a parallel double translation with a reconciliation procedure is the preferred approach (Hambleton \& Patsula, 1999; ITC, 2017; PISA, 2018).

Translation by a committee involves several bilingual people familiar with the tool domain and the target culture. Committee members may do an initial translation individually or work from one or two original translations (Caron, 1999; Lecavalier \& Tassé, 2001). The aim of the committee is to compare the different translations and to establish a consensus version. This technique protects against biases linked to a limited number of translators and makes it possible to verify whether the original version has been adapted to the target culture. It is recognized by many researchers for producing more accurate translations than individual translators (Hambleton \& Patsula, 1999). In addition to researchers and translators, it is recommended that the committee include a professional translator or linguist in the target language to ensure proper writing; experts in the field so as to use appropriate specialized terms; and, if possible, the author of the original tool to clarify ambiguities generated by translation (Caron, 1999; Daly, 2010; Vallerand, 1989). The rules for making decisions within the committee are as follows: the meaning of the original item takes precedence over literal translation; the properties of the language as spoken in the target environment must take priority; the accuracy of the technical terms in the target language must 
be ensured; in case of disagreement, alternative formulations must be submitted to users for pre-testing; finally, the presentation format and instructions for use of the translated tool must be the same as the original tool (Vallerand, 1989). Because of its advantages, this technique forms a necessary part of the methodology for cross-cultural validation of measurement tools (Vallerand, 1989).

Pre-testing of the draft version (also called the experimental version) is necessary to ensure that the tool items and instructions are clear, unambiguous, and in a language corresponding to that of the target users (Hambleton \& Patsula, 1999; ITC, 2017; WHO, n.d.). Two methods are common. The survey method with a sample of target users invites them to point out ambiguous items; those items that are reported repeatedly require reformulation (Lecavalier \& Tassé, 2001; Vallerand, 1989). The group interview method with subjects who are representative of the target users also makes it possible to verify whether their understanding of the content corresponds to the intended meaning. This involves asking participants to explain their understanding of each item; if they cannot answer clearly, with the intended meaning, the item is considered ambiguous. In both methods, the pretest should allow participants to clarify the nature of ambiguities and to gather suggestions or opinions on different formulations of certain items. This information is later used by the committee to reformulate problematic items (Caron, 1999; Daly, 2010; ITC, 2017; WHO, n.d.).

\section{Validation of Translated Measurement Tools}

The validation of translated tools is used to evaluate their metric properties, compare these properties with those of the original tool, and correct any translation and adaptation problems (Hambleton \& Patsula, 1999; ITC, 2017; Vallerand, 1989). Tests may concern the content validity, concurrent validity, construct validity, reliability of the translated tool, or the standards against which to assess its results (Lecavalier \& Tassé, 2001; Vallerand, 1989). It is up to the researchers to determine which tests are required based on the nature of the tool and the demonstrated metric properties of the original version. Small-scale studies with the target population are recommended in order to make any necessary revisions to the adapted tool, before proceeding with larger-scale empirical studies on the equivalence of instruments with respect to their metric properties (ITC, 2017).

\section{METHODOLOGY OF TRANSLATION AND CULTURAL ADAPTATION OF THE ORIGINAL FRENCH TOOL}

The methodology used in this study is based on the best practices of the abovementioned methodological guidelines. Two methods were used. First, translation by a committee of experts using parallel double translation was used to translate the tool and verify its equivalence with the original tool. These techniques are favoured by the authorities in the field. Their relevance lies in the group discussion that takes place around the content of the translation, which offers a certain guarantee against the biases of individual translators (Hambleton \& Patsula, 1999; ITC, 2017; PISA, 
2018). Second, a pretest of the preliminary English version was performed to adapt the tool to the culture of the target users. The group interview method was chosen because it met the main pretest requirement, which was to allow participants to explain the nature of the ambiguities they identify and their suggestions for correcting the tool (Caron, 1999; Daly, 2010; ITC, 2017; WHO, n.d.).

The expert committee members were all bilingual. There were four anglophones - two professional translators, a researcher, and a practitioner (the latter both experts in the field)-and the francophone researcher who authored the original tool. The expert committee had appropriate resources to perform an accurate translation: professional translators who were native speakers of the target language, to ensure the correct linguistic form in English; bilingual experts in the field who were familiar with terminology in both languages, to ensure that the specialized terms used were rigorously reviewed with reference to the theoretical content; and the author of the original tool, to reduce the risk of misunderstandings among committee members and to clarify ambiguities generated by the translation process (Caron, 1999; Daly, 2010; Vallerand, 1989). The role of the expert committee was to evaluate the translations, select the most appropriate wordings, reformulate as necessary, and ensure consistency with the meaning of the original tool. All decisions were made by consensus (always confirmed by vote) among committee members. This procedure led to a preliminary English version of the tool.

Target user adaptation of the tool was carried out by conducting a pretest of the preliminary English version through a focus-group interview with a sample of target users. The focus group was composed of eight participants from Toronto. All had relevant experience in partnership. Three were from public health (two departments) and five were from non-profit groups and community networks (with roles ranging from executive directors to community volunteers). They represented various fields, such as chronic disease prevention, food justice, children's health, women's health, community development, and anti-poverty. The purpose of the pretest was to ensure both that the tool's items were clear, understandable, and unambiguous, in a language corresponding to that of the target users, and that the users' understanding corresponded to the meaning of the original tool. Any item that did not meet these two criteria was reviewed for correction. During the pretest, participants were asked not only to point out ambiguous terms or items but also to provide information (explanations, rewordings) that could correct them. This information was subsequently used by the expert committee to reformulate the problematic items (Caron, 1999; Daly, 2010; Hambleton \& Patsula, 1999; ITC, 2017; WHO, n.d.). After a final review, the adapted tool was given final approval by the expert committee.

\section{RESULTS}

The translation-adaptation of the tool was conducted from December 2016 to December 2017. In preparation, a proposal for translation-adaptation was developed 


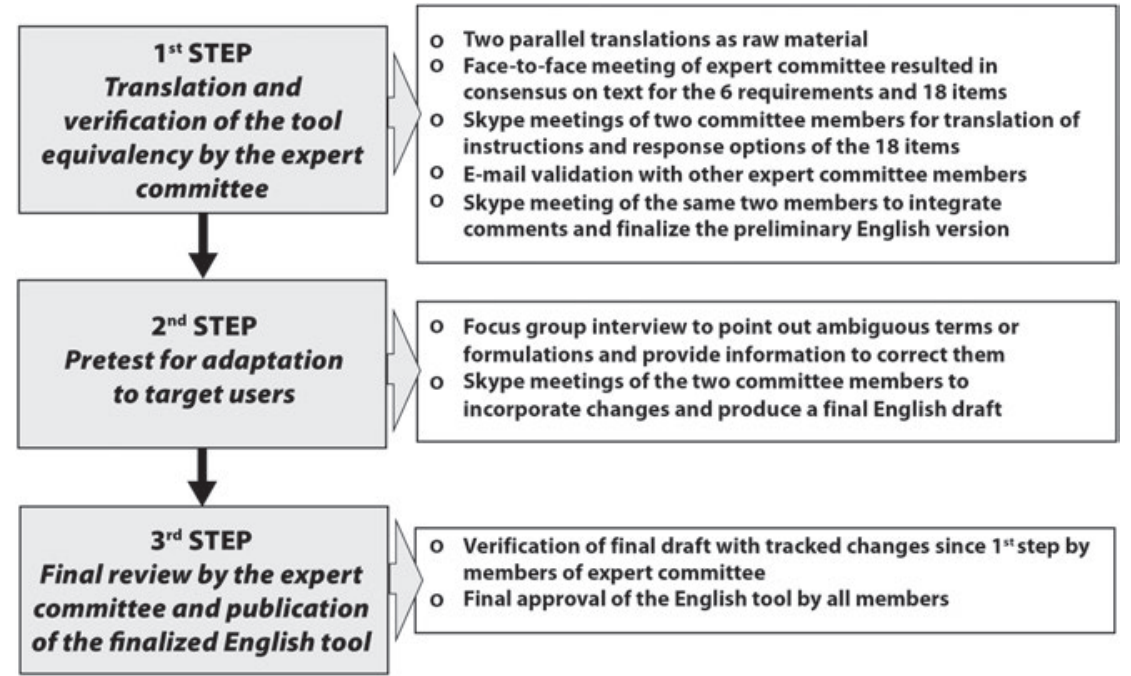

Figure 1: The three-step process of translation-adaptation of the original tool

and a partnership agreement was established between Health Nexus of Toronto and the researcher from l'Université de Montréal, who is the author of the original tool. The expert committee for translation was then established. The full process, jointly led by the bilingual expert practitioner (from Health Nexus) and the original tool author (from l'Université de Montréal), was carried out in three steps. The approach for each step is presented below, followed by the results. Figure 1 summarizes the approach for each step.

\section{Step 1: Translation of the Original French Tool and Verification of Its Equivalency by the Expert Committee-June 2017}

The tool has three sections: (1) the instructions, which cover the nature of the tool and the six requirements for effective partnership that are evaluated, the three ways to use the tool in a partnership and how to compile the results, and the three types of partnership evaluation that can be produced; (2) collection of information on the respondent (affiliation, role, and time in the partnership); and (3) the 18 items of the tool, with three response options for each.

To begin, two parallel translations of the tool were carried out independently by the committee's two professional translators. This resulted in two very distinct translations, reflecting the differences in the two translators' backgrounds. The first one works full time in translation, but not specifically within the field of the tool; the second one works full time within this field and does internal translation work for Health Nexus. The two translations were the raw material used by the expert committee to develop the preliminary version of the English tool. 
The committee edited the translation beginning with the tool's six requirements, then worked through the 18 items, followed by the three options under each item, and finally completed the instructions. This sequence enhanced the accuracy and consistency of the translated texts, from foundation to finish.

The bulk of this work was done in a three-hour face-to-face meeting between all five expert committee members. Both professional translations had been previously submitted to members, asking them to choose their favoured formulations, taking into consideration equivalency in meaning with the original tool, accuracy of English terms commonly used in the field, and clarity of language. At the meeting, a third translation, previously produced by a francophone bilingual professor from the École de santé publique of l'Université de Montréal (who uses the tool professionally), was also used, without the professor participating on the committee. Working together on one screen, the committee proceeded as follows: (1) committee members consulted a three-column table showing the three translations side by side; (2) for each piece (i.e., each requirement or each item), the academic expert first identified a favoured wording to start from; (3) then committee members identified terms and wording to deliberate on and together built a consensus on a preferred formulation; (4) correspondence with the meaning in the French tool was verified and, when needed, the committee went back to reformulate.

The meeting resulted in a consensual choice of texts for the six requirements and 18 items. For five of the 18 items, the committee retained the wording of one of the two professional translations. For the remaining 13 items, as well as the six requirements, the concluding formulation borrowed wording from one or more of the three translations and/or wording proposed by committee members.

The instructions (apart from the six requirements) and the three response options within each of the 18 items were selected and edited from the translated versions over the course of two Skype meetings (three hours each) between two committee members, the practitioner expert, and the author of the original tool. The procedure was the following: (1) while referencing the full committee's chosen formulation for an item, and its three response option translations, the most appropriate terms and wording were identified; (2) correspondence with the meaning in the French tool was then verified; (3) finally, the resulting formulations were validated by email with the remaining expert committee members.

Expert committee members proposed changes for eight of the 54 options (six single options under six separate items and two options under one single item) and four modifications to the instructions. The preliminary English version of the instructions underwent a few notable changes: instructions were spread over two pages instead of one, relying more on point form; and the English term evaluation was used to translate the French diagnostic, in accordance with the translated title of the tool. The procedure resulted in a consensual formulation of the three response options for each item, and of the instructions. A concluding Skype meeting between the practitioner expert and the author of the original tool was held to integrate all proposed changes and finalize the preliminary English version of the 
tool. This English version used the same presentation format and instructions as the original version (except for the two-page length of the instructions), since this condition contributes to the equivalence of the two versions (Vallerand, 1989).

\section{Step 2: Pretest of the Preliminary English Version Tool with a Sample of Target Users-July to October 2017}

A two-hour focus group interview was performed with the eight participants. The interview was led by the practitioner expert, with support staff assistance for note-taking. The author of the original tool observed the focus group through remote audio, in order to monitor when and how well participants' understanding corresponded to the meaning of the original tool.

In preparation for the pretest procedure, the practitioner expert asked participants to complete the tool individually by applying it to their partnership. At the beginning of the focus-group session, participants were asked to indicate, by marking on a poster-size version of the tool, any sections that they found difficult to understand. These notes served later as a cue for additional review. Then, for the instructions, as well as for each item and its three response options, the interviewer (1) invited one participant to explain the item in their own words; (2) checked whether other participants had a similar or different comprehension; and (3) for ambiguous statements, comprehension difficulties, or statements that were understood differently from the meaning in the original tool, invited participants to explain the ambiguities and suggest reformulations that improved clarity. Immediately following the focus group, the practitioner expert and the author of the original tool held a Skype meeting to analyze the results. Thereafter, two more Skype meetings were held to produce a final draft of the English translation that incorporated all changes resulting from the pretest. The pretest resulted in the adaptations described below.

\section{Adaptations to the Instructions}

Changes were made to the instructions section to clarify how to compile the results. Without changing the presentation of the tool, additional information was presented under a new title: Value of options. The instructions section was then resubmitted to the focus group participants by email in October to validate its clarity.

\section{Adaptations to Items and Response Options}

Adjustments were made to eight of the 18 items. In items 1, 2, and 3, issues and options for action was selected instead of problem and solution. Focus-group participants noted that issue is a more positive and broader term than problem and can reflect the range of situations that mobilize partnerships. Also, these terms better conveyed the original French meaning of problemes et solutions, which refer to the idea of the broad issues that mobilize partners. In items 5, 13, and 18, more precise wording was adopted. The guiding principle for decisions on such changes was that the choice of terms, as understood by the target users, should be informed 
Table 1. Adaptations to the tool's response options and basis for decisions

\begin{tabular}{ll}
\hline Response options & Basis of decisions \\
\hline Item 1, option 2 & Standardization \\
Item 3, option 3 & More common terms for target users \\
Item 4, options 1, 2,3 & Terms/wording closer to the meaning of the original tool \\
Item 6, option 2 & Standardization \\
Item 8, options 1, 3 & Terms/wording closer to the meaning of the original tool \\
Item 13, options 1, 2,3 & Standardization \\
Item 14, options 1, 2,3 & More common terms for target users \\
Item 15, option 3 & Terms/wording closer to the meaning of the original tool \\
\hline
\end{tabular}

by the meaning of the original tool rather than by literal translation (Caron, 1999; Vallerand, 1989). Moreover, in item 2, the term population was replaced by people. Also, item 14 was reworded to be more direct. For these two items, the guiding principle was to take into account the Canadian English language as spoken by the target users (Vallerand, 1989). There was one instance where the focus group participants identified a translation problem but no acceptable solution. For item 17, they suggested the use of the term collective impact to translate solutions intégrées and shared a strong consensus on this proposed change. While popular in current discourse on partnership, the term collective impact did not accurately translate the meaning, from the original tool, of solutions intégrées (which has a broader scope). Instead, the term holistic was proposed by the researcher-author and later approved by the full expert committee.

Adaptations were also made to the response options for eight of the items. For five items, these adaptations were made for the same reasons as described above. For three items, the changes were aimed at standardizing terminology used, either between response options or in reference to the item statement. These results are detailed in Table 1.

\section{Step 3: Final Review by the Expert Committee and Publication of the Completed English Tool-November 2017}

The resulting final draft of the English tool, with changes tracked since the preliminary English version, was submitted by email to the three other members of the expert committee. These members approved all changes resulting from the pretest, but they added four minor corrections, word changes that refined the adaptation in item 3 (option 2), item 5, item 8 (option 1), and item 15. All committee members approved publication of this final English tool.

\section{DISCUSSION}

The methodology used in this study-combining an expert committee process with two parallel translations and a pretest with target users-lends the approach 
a high degree of rigour. At each step, this methodology led to improvements in the Canadian English tool. The pretest mainly facilitated changes to more common terms, from the point of view of the target users, and rewordings for greater clarity or a better match with the original tool. The pretest did not result in any major cultural adaptations, reflecting the proximity between the socio-cultural environment of Quebec and English Canada in this field. Through an initial half-day face-to-face meeting of the committee, Skype meetings between the two coordinating committee members, and regular email communications among all members, the expert committee worked effectively to build consensus on the best words and sentence formulations for the three sections of the tool. The rigour of the methodology ensured equivalence of the translated tool with the original tool and solid cultural adaptation to the targeted context (Caron, 1999; Vallerand, 1989; Vézina, Samson-Morasse, Gauthier-Desgagné, Fossard, \& Sylvestre, 2011).

When it is empirically demonstrated that a translated version is closely linked to the original version, this supports attribution of the same content validity of the original tool to the translated tool (Vallerand, 1989). Ensuring that items are formulated in equivalent specialized terms, understandable, and culturally appropriate for users is a way to ensure the validity of the information provided by a tool (Ouellet, 2008). Using a committee of experts comprising not only translators but also a researcher and a practitioner familiar with the terminology in both languages, as well as the researcher who produced the original tool, allows us to argue convincingly that the specialized terms used in the English version correspond very closely to the terms and theoretical content of the original tool. During the pretest process, verifying that the target users' comprehension matched the meaning of the items in the original tool was another methodological element that supports the content validity of the translated tool. The construct validity of the translated tool, namely its ability to capture variations in respondents' judgment on the degree to which conditions have been met, to capture expected intra-partnership correlations, and to establish differences between partnerships, will have to be confirmed in a later study.

\section{CONCLUSION}

The methodology used was a rigorous process wherein the new version of the tool was adjusted through evaluations by various experts and target users in the field. This ensured that it corresponded to the meaning of the French tool and that it was culturally adapted to its intended users. Of further value, the tool includes indicators that concern a partnership's capacity to direct its multiple stakeholder perspectives towards innovation and includes important process equity indicators that have been raised within many partnership circles in public health. The resulting Self-evaluation tool for action in partnership responds well to the evaluation needs of partnerships within English-speaking Canada and other comparable socio-cultural English-speaking regions. 


\section{ACKNOWLEDGMENTS}

Health Nexus acknowledges the financial support of Status of Women Canada that made this project possible. The authors would like to thank the members who graciously agreed to participate in the expert committee: Robyn Kalda (Health Nexus), Rosalind Lobo (Traductions À la page-translation agency), and Suzanne F. Jackson (University of Toronto, Dalla Lana School of Public Health). They also thank all participants of the focus group: Anonymous (community volunteer), Denise Earle (community volunteer), Elaine Ebach (Toronto Public Health), Julie Maher (Ontario Women's Health Network), Tracey Methven (Toronto Public Health), Talisha Ramsaroop (Social Planning Toronto), Patricia Stevens (Toronto Public Health), and Barbara Willet (Health Nexus). The translation of this article has benefited from the financial support of the REGARDS Team (Fonds de recherche du Québec-Société et culture, 2015-SE-179156) at InterActions-Centre de recherche et de partage des savoirs, CIUSSS du Nord-de-l'île-de-Montréal. The authors also wish to express their thanks to Patrick Riley for proofreading this article.

\section{NOTE}

1 Available at https://en.healthnexus.ca/sites/en.healthnexus.ca/files/resources/selfevaluationtool.pdf

\section{ORCID ID}

Angèle Bilodeau (D) 0000-0002-4400-8624

\section{REFERENCES}

Auspos, P., \& Kubisch, A. C. (2004). Building knowledge about community change: Moving beyond evaluations. New York, NY: The Aspen Institute. Retrieved from https:// assets.aspeninstitute.org/content/uploads/files/content/docs/rcc/BUILDINGKNOWELDGE.pdf

Barnes, M., Matka, E., \& Sullivan, H. (2003). Evidence, understanding and complexity: Evaluation in non-linear systems. Evaluation, 9(3), 265-284. https://doi. org/10.1177/13563890030093003

Bilodeau, A., Galarneau, M., Fournier, M., \& Potvin, L. (2011). L'Outil diagnostique de l'action en partenariat: Fondements, élaboration et validation. Revue canadienne de santé publique, 102(4), 298-302. Retrieved from https://link.springer.com/ article/10.1007\%2FBF03404054

Brewer, M. (2000). Research design and issues of validity. In H. Reis \& C. Judd (Eds.), Handbook of research methods in social and personality psychology (pp. 3-17). Cambridge, England: Cambridge University Press.

Caron, J. (1999). Un guide de validation transculturelle des instruments de mesure en santé mentale. Rouyn, QC: Université du Québec en Abitibi-Témiscamingue. Retrieved from http://instrumentspsychometriques.mcgill.ca/instruments/guide.htm 
Chircop, A., Bassett, R., \& Tatlor, E. (2015). Evidence on how to practice intersectoral collaboration for health equity: A scoping review. Critical Public Health, 25(2), 178-191. https://doi.org/10.1080/09581596.2014.887831

Dahler-Larsen, P. (2001). From programme theory to constructivism: On tragic, magic and competing programmes. Evaluation, 7(3), 331-350. https://doi. org/10.1177/13563890122209711

Daly, Y. (2010). L'adaptation transculturelle d'une échelle de mesure : Cas de l'échelle des ancres de carrière / The transcultural adaptation of a measure scale: Case of the career anchors scale. Psychologie du travail et des organisations, 16(2), 160-176. https://doi. org/10.1016/S1420-2530(16)30154-6

Devaux-Spatarakis, A. (2014). Lévaluation "basée sur la théorie," entre rigueur scientifique et contexte politique. Politiques \& Management Public, 31(1), 51-68. https://doi.org/ 10.3166/pmp.31.51-68

Fetterman, D., \& Wandersman, A. (2007). Empowerment evaluation: Yesterday, today, and tomorrow. American Journal of Evaluation, 28(2), 179-198. https://doi. org/10.1177/1098214007301350

Halliday, J., Asthana, S. N. M., \& Richardson, S. (2004). Evaluating partnership: The role of formal assessment tools. Evaluation, 10(3), 285-303. https://doi. org/10.1177/1356389004048279

Hambleton, R. K., \& Patsula, L. (1999, August). Increasing the validity of adapted tests: Myths to be avoided and guidelines for improving test adaptation practices. Journal of Applied Testing Technology, 1-12. Retrieved from http://www.jattjournal.com/index. php/atp/article/view/48345/39215

International Test Commission (ITC). (2017). ITC guidelines for translating and adapting tests (second edition). International Journal of Testing, 18(2), 101-134. https://doi.org /10.1080/15305058.2017.1398166

Lecavalier, L., \& Tassé, M. J. (2001). Traduction et adaptation transculturelle du Reiss screen for maladaptive behavior. Revue francophone de la déficience intellectuelle, 12(1), 31-44. Retrieved from http://www.rfdi.org/wp-content/uploads/2013/05/ LECAVALIER_v12.pdf

Newcomer, K. E., Hatry, H. P., \& Wholey, J. S. (2015). Handbook of practical program evaluation (4th ed.). Hoboken, NJ: John Wiley \& Sons.

Ouellet, S. (2008). Adaptation et validation d'un instrument de mesure des besoins et des attentes des aidants familiaux en soins palliatifs. Revue de l'Université de Moncton, 39(1-2), 97-127. https://doi.org/10.7202/039844ar

Presser, S., Couper, M. P., Lessler, J. T., Martin, E., Martin, J., Rothgeb, J. M., \& Singer, E. (2004). Methods for testing and evaluating survey questions. In S. Presser, J. M. Rothgeb, M. P. Couper, J. T. Lessler, E. Martin, J. Martin, \& E. Singer (Eds.), Methods for testing and evaluating survey questionnaires (pp. 1-22). Hoboken, NJ: John Wiley \& Sons.

Programme for International Student Assessment (PISA). (2018). PISA 2018 translation and adaptation guidelines. Prague, Czech Republic: OECD. Retrieved from https://www. oecd.org/pisa/pisaproducts/PISA-2018-translation-and-adaptation-guidelines.pdf 
Vallerand, R. J. (1989). Vers une méthodologie de validation trans-culturelle de questionnaires psychologiques : implications pour la recherche en langue française. Canadian Psychology / Psychologie canadienne, 30(4), 662-680. https://doi.org/10.1037/ h0079856

Vézina, M., Samson-Morasse, C., Gauthier-Desgagné, J., Fossard, M., \& Sylvestre, A. (2011). Développement de la version québécoise francophone du Children's Communication Checklist-2 (CCC-2). Traduction, adaptation et équivalence conceptuelle. / Development of a Quebec French version of the Children's Communication Checklist-2 (CCC-2). Translation, adaptation and conceptual equivalence. Revue canadienne d'orthophonie et d'audiologie (RCOA), 35(3), 244-253.

Villeval, M., Bidault, E., Shoveller, J., Alias, F., Basson, J. C., Frasse, C., .. \& \& Lang, T. (2016). Enabling the transferability of complex interventions: Exploring the combination of an intervention's key functions and implementation. International Journal of Public Health, 61(9), 1031-1038. https://doi.org/10.1007/s00038-016-0809-9

World Health Organization (WHO). (2011). Intersectoral action on health: A path for policy-makers to implement effective and sustainable action on health. Kobe, Japan: The WHO Center for Health Development. Retrieved from http://www.who.int/ kobe_centre/publications/ISA-booklet_WKC-AUG2011.pdf

World Health Organization (WHO). (n.d.). Process of translation and adaptation of instruments. Retrieved from http://www.who.int/substance_abuse/research_tools/ translation/en/

\section{AUTHOR INFORMATION}

Angèle Bilodeau holds a Ph.D. in applied human sciences and a master's in sociology. She is a full researcher/professor in the School of Public Health at l'Université de Montréal. She is also a researcher at the Centre InterActions, CIUSSS du Nord-de-l'île-de-Montréal, and a Canada Research Chair in Approches communautaires et inégalités de santé (CACIS). Her research contributions include intersectoral collaborations and governance, action in partnership and social innovation, and effects of intersectoral action on local neighbourhoods.

Gillian Kranias holds a Master in Environmental Studies and a BA in science and human affairs. She specializes in community development and intersectoral partnerships for healthy and equitable communities. She currently works as a bilingual health promotion consultant at Health Nexus, facilitating knowledge mobilization, evaluation, and strategic planning with partnerships. 